\title{
Threshold changeable secret image sharing using bivariate polynomial
}

\section{CURRENT STATUS: UNDER REVIEW}

EURASIP Journal on Image and Video Processing Springer

Yanxiao Liu

XI'AN University of Technology

liuyanxiao@xaut.edu.cnCorresponding Author

ORCiD: https://orcid.org/0000-0002-2507-5143

Ping Wu

XI'AN University of Technology

Qindong Sun

XI'AN University of Technology

Zhili Zhou

Nanjing University of Information Science and Technology

DOI:

10.21203/rs.3.rs-16037/v1

SUBJECT AREAS

Nuclear Medicine \& Medical Imaging

KEYWORDS

secret image sharing, threshold changeable, bivariate polynomial 


\section{Abstract}

In secret image sharing (SIS) scheme, a confidential image is en-crypted into multiple shadows, any group of shadows that reaches the thresh-old, otherwise nothing can be reconstructed at all. Most existing SIS schemes have a fixed threshold, however in this work, we consider more complicat-ed cases that the threshold may be adjusted due to the changeable security environment. In this paper, we construct a ( $k \leftrightarrow h, n)$ threshold changeable SIS (TCSIS) scheme using bivariate polynomial, which has $\mathrm{h}-\mathrm{k}+1$ possible thresholds $\mathrm{k}, \mathrm{k}+1, \ldots, \mathrm{h}$. During image reconstruction, each participant can update the his shadow according to the current threshold T only based on his initial shadow.

Comparing with previous TCSIS schemes, the proposed scheme achieves unconditional security, and can overcome the information disclosure problem caused by homomorphism.

\section{Full Text}

Due to technical limitations, full-text HTML conversion of this manuscript could not be completed. However, the manuscript can be downloaded and accessed as a PDF.

\section{Figures}

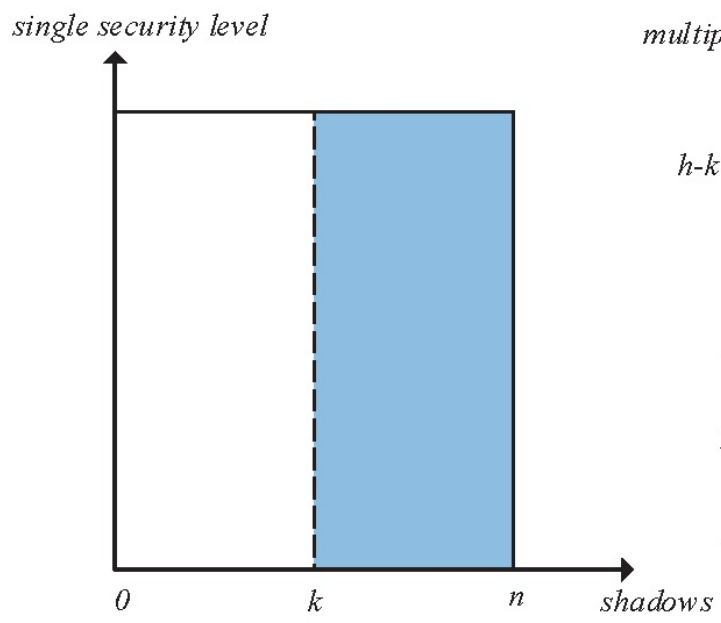

(a)

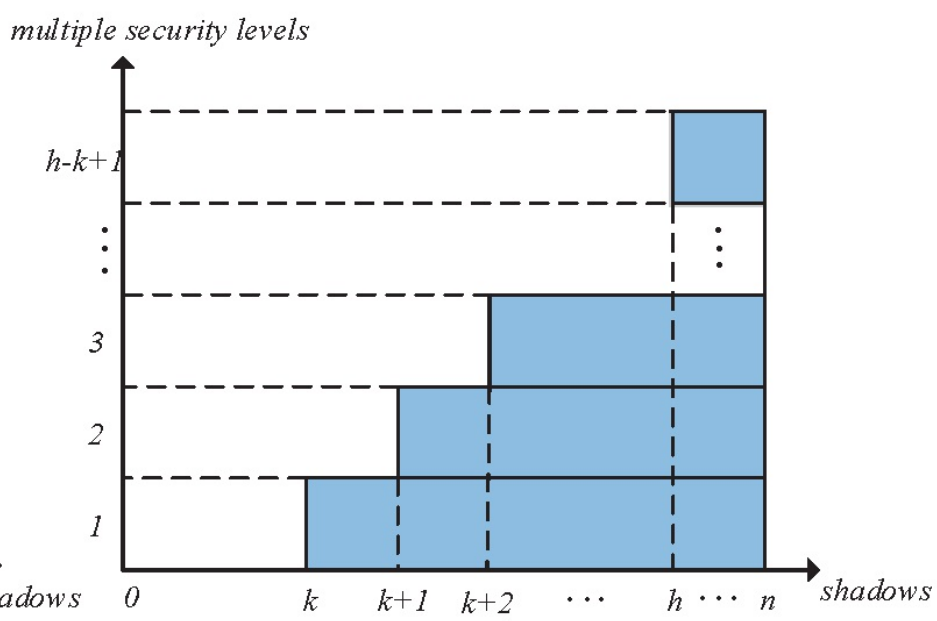

(b)

Figure 1

Thresholds for different schemes: $(a)(k, n)$ SIS scheme (b) $(k<-->h, n)$ TCSIS scheme 


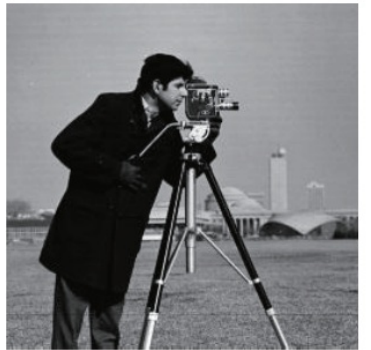

original image

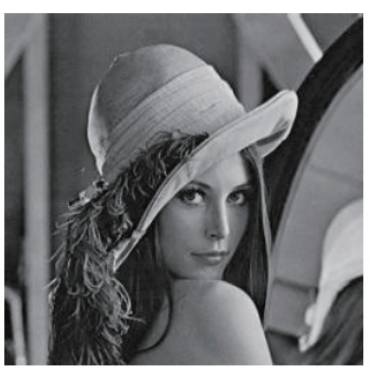

original image

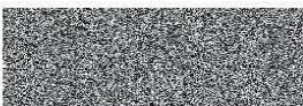

1

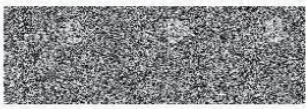

4

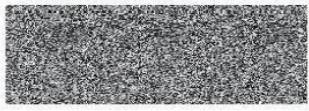

7

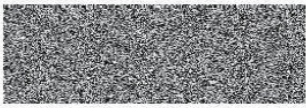

1

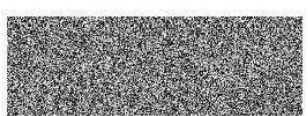

4

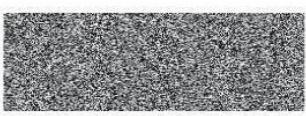

7

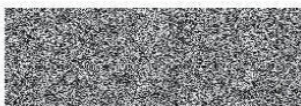

2

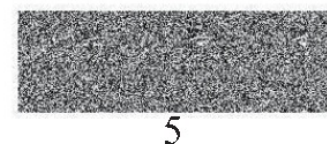

5

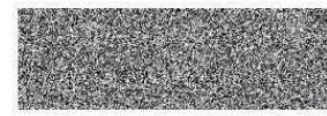

8

shadows

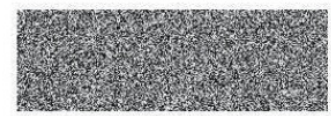

2

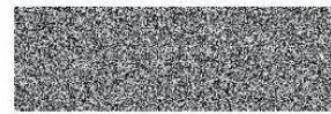

5

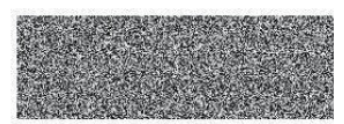

8

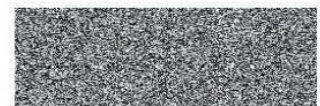

3

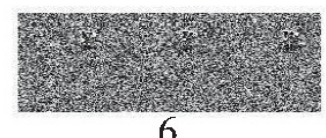

6

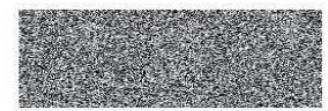

9

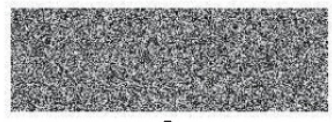

3

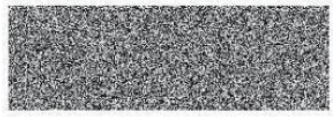

6

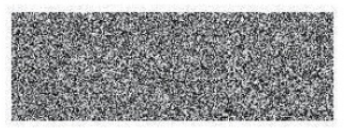

9

shadows

Figure 2

Original images and shadows using proposed scheme 


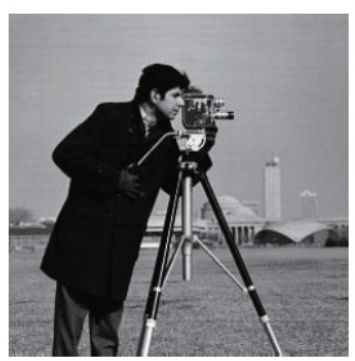

$a-1$
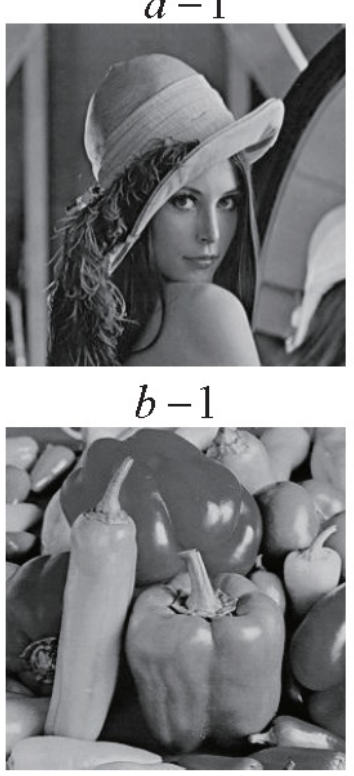

$c-1$

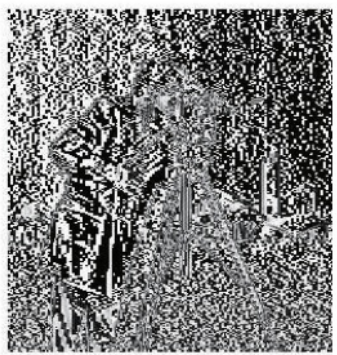

$a-2$

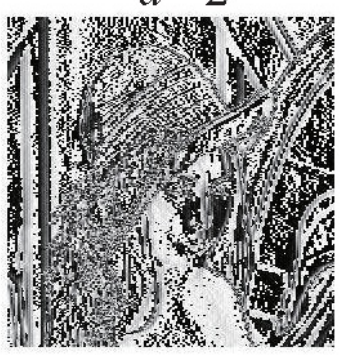

$b-2$

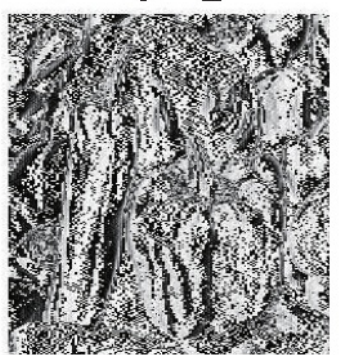

$c-2$

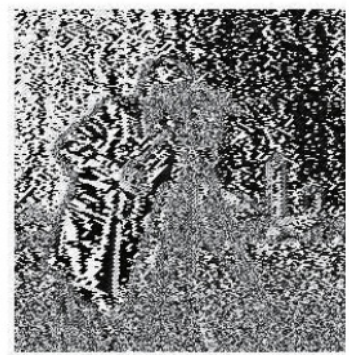

$a-3$

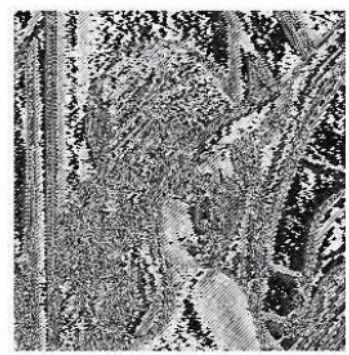

$b-3$

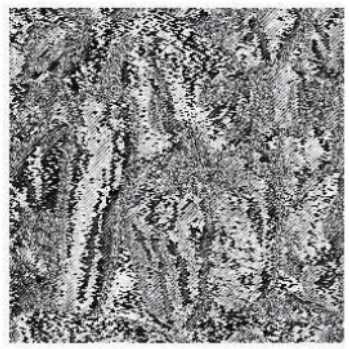

$c-3$

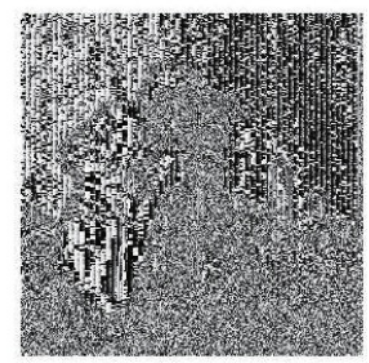

$a-4$
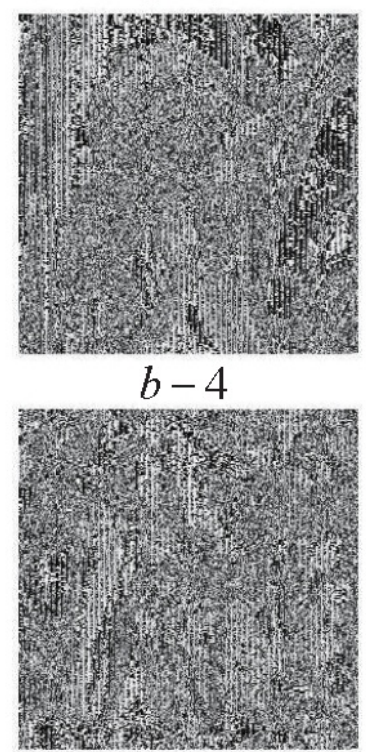

$c-4$

Figure 3

$(a-1, b-1, c-1)$ : original images, $(a-2, b-2, c-2)$ : quality lossy images with $\left(k^{\prime}=2 ; k=3\right),(a-3, b-$ 3,c-3): quality lossy images with $\left(k^{\prime}=3 ; k=4\right),(a-4, b-4, c-4)$ : quality lossy images with $\left(k^{\prime}=\right.$ $4 ; k=5)$

\section{Supplementary Files}

This is a list of supplementary files associated with this preprint. Click to download.

\section{SI-Liu.tex}

